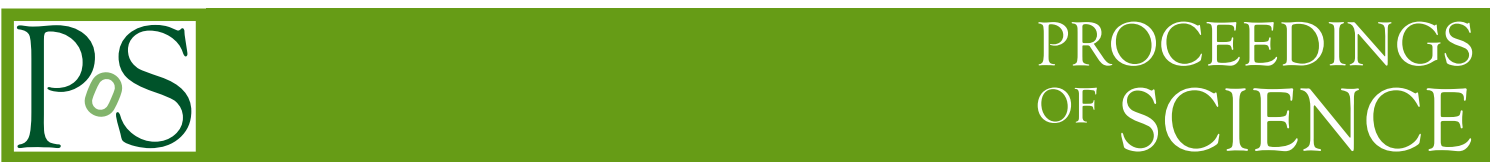

\title{
Healthy theories beyond Horndeski
}

\section{Filippo Vernizzi*}

Institut de Physique Théorique - CEA

E-mail: filippo.vernizzi@cea.fr

I will present a new class of scalar-tensor theories that extend Horndeski theories. Despite possessing equations of motion of higher order in derivatives, their true propagating degrees of freedom obey well-behaved second-order equations and are thus free from Ostrogradski instabilities, in contrast to the standard lore. Remarkably, the covariant versions of the original galileon Lagrangians obtained by direct replacement of derivatives with covariant derivatives belong to this class of theories. These extensions of Horndeski theories exhibit an uncommon, interesting phenomenology: the scalar degree of freedom affects the speed of sound of matter, even when the latter is minimally coupled to gravity.

Frontiers of Fundamental Physics 14 - FFP14,

15-18 July 2014

Aix Marseille University (AMU) Saint-Charles Campus, Marseille

\footnotetext{
* Speaker.
} 